\title{
Correction to: MLIC: A MaxSAT-Based Framework for Learning Interpretable Classification Rules
}

Dmitry Malioutov and Kuldeep S. Meel

\begin{abstract}
Correction to:
Chapter "MLIC: A MaxSAT-Based Framework for Learning Interpretable Classification Rules" in: J. Hooker (Ed.): Principles and Practice of Constraint Programming, LNCS 11008, https://doi.org/10.1007/978-3-319-98334-9_21
\end{abstract}

In the original version of this paper there was a typing error in the family name of the first author. "Dmitry Maliotov" should have been "Dmitry Malioutov". This has now been corrected.

The updated version of this chapter can be found at https://doi.org/10.1007/978-3-319-98334-9_21 